\section{New Necessary Stability Conditions for 2-D Systems}

\author{
Nikos E. Mastorakis
}

Abstract-In this paper, some interesting necessary conditions for the stability of two-dimensional (2-D) systems are presented. The inversion of these conditions gives sufficient conditions for the instability of the same systems. The proof of these conditions is given. A comparison with some other known criteria is given.

Index Terms-Multidimensional systems, stability, stability test, two-dimensional system.

\section{INTRODUCTION}

Two-dimensional (2-D) systems theory has recently attracted increasing attention in the areas of analysis, synthesis, stability, factorizability, controllability, observability, minimality, feedback control, and filter design.

Stability of 2-D systems arises in many applications. Two-dimensional signal processing, realization of 2-D networks and distributed parameter systems, stability of 2-D difference/differential equations, processing of radar data are among these applications.

In [1], an excellent overview of the stability problem and of the theorems and the tests associated with it has been made by Jury. Some of these theorems check if some one-dimensional (1-D) or 2-D polynomials is devoid of zeros in appropriate regions of $C$ or $C x C$, respectively. For this reason, the factorization problem of 2-D polynomials is all important [3]-[6].

In the literature, several tests exist which check various conditions of the coefficients of $f\left(z_{1}, z_{2}\right)$ [1], [6]-[8]. From another standpoint, we obtain that most of tests check sufficient conditions for stability [6]-[14], while other tests check necessary stability conditions [15], [17]. The inversion of the latter conditions gives sufficient conditions for instability. Other recent relevant studies can be found in [18]-[22].

A shift-invariant causal single-input single-output (SISO) 2-D system can be described by the transfer function

$$
G\left(z_{1}, z_{2}\right)=\frac{g\left(z_{1}, z_{2}\right)}{f\left(z_{1}, z_{2}\right)}
$$

where $g\left(z_{1}, z_{2}\right)$ and $f\left(z_{1}, z_{2}\right)$ are coprime polynomials in the independent complex variables $z_{1}$ and $z_{2}$, It is assumed that there are no nonessential singularities of the second kind on the closed unit bidisk [1], i.e., there are no points $\left(z_{1}, z_{2}\right)$ with $\left|z_{1}\right| \leq 1$ and $\left|z_{2}\right| \leq 1$ such that $g\left(z_{1}, z_{2}\right)=f\left(z_{1}, z_{2}\right)=0$.

It is well known that the system (1) is BIBO stable, if and only if

$$
\begin{array}{rlrl}
f\left(z_{1}, 0\right) & \neq 0, & & \left|z_{1}\right| \leq 1 \\
f\left(z_{1}, z_{2}\right) \neq 0, & & \left|z_{1}\right|=1, \quad\left|z_{2}\right| \leq 1
\end{array}
$$

Condition (2a) is relatively easy to check using any 1-D stability test. Checking (2b) is a more difficult task.

In the present paper, some interesting necessary conditions for 2-D system stability are presented. The inversion of these conditions gives sufficient conditions for 2-D systems instability. These can be used as

Manuscript received January 26, 1999; revised November 23, 1999. This paper was recommended by Associate Editor J. Götze.

The author is with the Military Institutions of University Education, Hellenic Naval Academy, Terma Hatzikyriakou, 18539 Piraeus, Greece.

Publisher Item Identifier S 1057-7122(00)05501-X. a quick check if the 2-D system is unstable. The main result is given by Theorem 1 . Some numerical examples are also included via which a comparison with other necessary stability conditions is given.

\section{MAIN REsUltS}

Consider the 1-D polynomial

$$
f(z)=\sum_{i=0}^{N} \alpha_{f}(i) \cdot z^{i} \quad \text { with } \alpha_{f}(i), z \in C .
$$

The reciprocal (or reverse) polynomial is defined as the reversed and conjugated one.

$$
\bar{f}(z)=\sum_{i=0}^{N} \alpha_{f}^{*}(N-i) \cdot z^{i}
$$

or in a more convenient notation

$$
\bar{f}(z)=\sum_{i=0}^{N} \alpha_{\bar{f}}(i) z^{i} \quad \text { with } \alpha_{\bar{f}}(i)=\alpha_{f}^{*}(N-i)
$$

where $*$ means the complex conjugate. The partial energy of the polynomial $f(z)$ is defined as

$$
\operatorname{PE}_{f}(n)=\sum_{i=0}^{n}\left|\alpha_{f}(i)\right|^{2}, \quad n=0,1, \ldots, N-1
$$

while the total energy is defined by

$$
\operatorname{TE}_{f}(N)=\sum_{i=0}^{N}\left|\alpha_{f}(i)\right|^{2} .
$$

Note that

$$
\operatorname{TE}_{f}(N)=\operatorname{TE}_{\bar{f}}(N) .
$$

If the polynomial $f(z)$ corresponds to a stable 1-D system, i.e., it is the characteristic polynomial of a stable 1-D system, then

$$
\operatorname{PE}_{f}(n)>\operatorname{PE}_{\bar{f}}(n) \quad \forall n, n=0,1, \ldots, N-1 .
$$

Relations (9) express the so-called minimal delay property. That means that the stable polynomial $f(x)$ has most of its energy concentrated at the earlier times, while $\bar{f}(z)$ has most of its energy concentrated at the later times. A detailed proof is given in [2]. In fact, equations (9) are necessary stability conditions and therefore they can be used as a quick check if the 1-D system is unstable.

In order to extend the above ideas to the 2-D case, let us consider the 2-D polynomial

$$
f\left(z_{1}, z_{2}\right)=\sum_{i_{1}=0}^{N_{1}} \sum_{i_{2}=0}^{N_{2}} \alpha_{f}\left(i_{1}, i_{2}\right) z_{1}^{i_{1}} z_{2}^{i_{2}}
$$

with $\alpha_{f}\left(i_{1}, i_{2}\right), z_{1}, z_{2} \in C$.

It should be noted that in 2-D circuits, 2-D filters, and other engineering applications, we have $\alpha_{f}\left(i_{1}, i_{2}\right) \in \boldsymbol{R}$.

The reciprocal (reverse) polynomial, with respect to $z_{2}$, is defined as

$$
\bar{f}\left(z_{1}, z_{2}\right)=\sum_{i_{1}=0}^{N_{1}} \sum_{i_{2}=0}^{N_{2}} \alpha_{f}^{*}\left(i_{1}, N_{2}-i_{2}\right) z_{1}^{i_{1}} z_{2}^{i_{2}}
$$


or

$$
\bar{f}\left(z_{1}, z_{2}\right)=\sum_{i_{1}=0}^{N_{2}} \sum_{i_{2}=0}^{N_{2}} \alpha_{\bar{f}}\left(i_{1}, i_{2}\right) z_{1}^{i_{1}} z_{2}^{i_{2}}
$$

with $\alpha_{\bar{f}}\left(i_{1}, i_{2}\right)=\alpha_{f}^{*}\left(i_{1}, N_{2}-i_{2}\right), i_{1}=0,1, \cdots, N_{1}$ and $i_{2}=$ $0,1, \cdots, N_{2}$. The reciprocal polynomial, with respect to $z_{1}$, is defined analogously.

The partial energy $\operatorname{PE}_{f}\left(n_{2}\right)$ and the total energy $\operatorname{TE}_{f}\left(N_{2}\right)$ can be defined as follows:

$$
\mathrm{PE}_{f}\left(n_{2}\right)=\sum_{i_{2}=0}^{n_{2}}\left|\left(\sum_{i_{1}=0}^{N_{1}} \alpha_{f}\left(i_{1}, i_{2}\right) z_{1}^{i_{1}}\right)\right|^{2}
$$

with $0 \leq n_{2}<N_{2}$,

$$
\operatorname{TE}_{f}\left(N_{2}\right)=\sum_{i_{2}=0}^{N_{2}}\left|\left(\sum_{i_{1}=0}^{N_{1}} \alpha_{f}\left(i_{1}, i_{2}\right) z_{1}^{i_{1}}\right)\right|^{2}
$$

Obviously

$$
\operatorname{TE}_{f}\left(N_{2}\right)=\operatorname{TE}_{\bar{f}}\left(N_{2}\right) \quad \text { with }\left|z_{1}\right|=1 .
$$

Now, the following theorem is stated.

Theorem 1: If the polynomial $f\left(z_{1}, z_{2}\right)$ corresponds to a stable 2-D system, i.e., it is the characteristic polynomial of a BIBO stable 2-D system (1), then

$$
\begin{array}{r}
\operatorname{PE}_{f}\left(n_{2}\right)>\operatorname{PE}_{\bar{f}}\left(n_{2}\right) \quad \text { with }\left|z_{1}\right|=1, \\
\forall n_{2}, n_{2}=0,1, \ldots, N_{2} .
\end{array}
$$

Proof: It follows directly from (2b).

Q.E.D.

Furthermore, in our analysis we assume that $a_{f}\left(i_{1}, i_{2}\right) \in R$. Then from (16), after some algebraic manipulation, one finds the equivalent $N_{2}$ relations

$$
\mathbf{a}_{(i)}^{t} \cdot \mathbf{c}>0 \quad \text { where } i=1, \ldots, N_{2}
$$

where $\mathbf{a}_{(i)}^{t}$ is a row-vector with $j$ th element, where $j=1, \cdots, N_{1}+$ $1, i=1, \cdots, N_{2}$

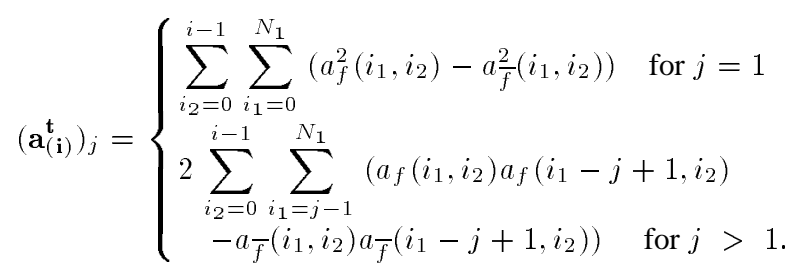

$\boldsymbol{c}$ is a column vector with $c_{j}=\cos ((j-1) \theta)$. Furthermore, using the following transformation:

$$
\begin{aligned}
\cos (j \theta)= & \frac{1}{2}\left\{(2 \cos \theta)^{j}-\frac{j}{1}(2 \cos \theta)^{j-2}\right. \\
& +\frac{j}{2}\left(\begin{array}{c}
j-3 \\
1
\end{array}\right)(2 \cos \theta)^{j-4} \\
& \left.-\frac{j}{3}\left(\begin{array}{c}
j-4 \\
2
\end{array}\right)(2 \cos \theta)^{j-6}+\ldots\right\}
\end{aligned}
$$

one can write

$$
c=T \cdot x
$$

where $x^{t}=\left[1 x x^{2}, \cdots, x^{N_{1}}\right]$ with $x=\cos \theta$. For example

$$
\begin{aligned}
{\left[\begin{array}{c}
1 \\
\cos \theta \\
\cos 2 \theta \\
\cos 3 \theta \\
\cos 4 \theta \\
\cos 5 \theta
\end{array}\right]=} & {\left[\begin{array}{cccccc}
1 & 0 & 0 & 0 & 0 & 0 \\
0 & 1 & 0 & 0 & 0 & 0 \\
-1 & 0 & 2 & 0 & 0 & 0 \\
0 & -3 & 0 & 4 & 0 & 0 \\
1 & 0 & -8 & 0 & 8 & 0 \\
0 & 5 & 0 & -20 & 0 & 16
\end{array}\right] } \\
& {\left[\begin{array}{c}
1 \\
\cos \theta \\
\cos ^{2} \theta \\
\cos ^{3} \theta \\
\cos ^{4} \theta \\
\cos ^{5} \theta
\end{array}\right] . }
\end{aligned}
$$

Therefore, from (17) one finds

$$
\mathbf{a}_{(i)}^{t} \cdot \mathbf{T} \cdot \mathbf{x}>0 \quad \text { where } i=1, \ldots, N_{2}
$$

or

$$
F_{1}(x)>0, \quad F_{2}(x)>0, \ldots, F_{N_{2}}(x)>0
$$

where $F_{i}(x)=\mathbf{a}_{(i)}^{t} \cdot \mathbf{T} \cdot \mathbf{x}$ is a polynomial in $x$ and $-1 \leq x \leq 1$. In order to check this local positivity of the above polynomials, we recall that a polynomial $F(x)$ is positive for $-1 \leq x \leq 1$ when the following two conditions are satisfied:

1. for an arbitrary $x_{0} \in[-1,1], F\left(x_{0}\right)>0$;

2. $F(x)$ is devoid of zeros in $-1 \leq x \leq 1$.

As a conclusion, Theorem 1, provides us a set of $N_{2}$ necessary conditions for 2-D systems stability. As sufficient conditions for instability, one can use

$\exists x_{0} \in[-1,1]$ and $\exists i, i=1, \ldots, N_{2}$ such that $F_{i}\left(x_{0}\right) \leq 0$.

1) Remark: It is clear that conditions analogous to (16)-(18) and (22)-(24) can be obtained by interchanging $z_{1}$ with $z_{2}$.

\section{EXAMPLES}

Example 1: Suppose that

$$
f\left(z_{1}, z_{2}\right)=1.56-2 z_{1}+z_{1}^{2}-0.5 z_{2}+z_{1} z_{2}-z_{2}^{2} .
$$

The 1-D condition $f\left(z_{1}, 0\right) \neq 0$ or $1.56-2 z_{1}+z_{1}^{2} \neq 0 \forall z_{1},\left|z_{1}\right| \leq 1$ is checked by an 1-D test or by finding the roots $z_{1}=-1 \pm 0.7843 i$. Then, the condition $\operatorname{PE}_{f}(0)>\mathrm{PE}_{\bar{f}}(0)$ is examined with $\left|z_{1}\right|=1$ or $\mathbf{a}_{(1)}^{t} \cdot \mathbf{T} \cdot \mathbf{x}=3.3136-10.24 x+6.24 x^{2}>0 \forall x:-1 \leq x \leq 1$. The roots of this polynomial are 0.443 and 1.198. So, for $x=0.5$ the polynomial is negative. Therefore, the corresponding 2-D system (1) is unstable. In fact, we have $f(0.5,0.9)=0$ which assures instability.

However, for this example, the method of [17] does not give a result, since one finds that both the zeros of $f(z, z)=1.56-2.5 z+z^{2}$ are outside the unit disk. As well, neither the zeros of $f_{1}(z, z)=$ $z^{N_{2}-1} f(z, 1 / z)=-1-0.5 z+2.56 z^{2}-2 z^{3}+z^{4}$ nor those of $f_{2}(z, z)=z^{N_{1}-1} f(1 / z, z)=1-2 z+2.56 z^{2}-0.5 z^{3}-z^{4}$ are located entirely inside or outside the unit disk. The zeros of $f_{1}(z, z)$ are $-0.4575,0.7405 \pm 1.3 i, 0.9766$, whereas the zeros of $f_{2}(z, z)$ are $-2.1857,0.3308 \pm 0.5808 i, 1.024$. Note also that the 1-D condition $f\left(0, z_{2}\right) \neq 0$ or $1.56-0.5 z_{2}+z_{2}^{2} \neq 0 \forall z_{2},\left|z_{2}\right| \leq 1$ is verified.

The method of [15, Theorem 3] cannot prove that this polynomial is unstable. More specifically, no inequality of this theorem is satisfied.

Example 2: This example is taken from [17].

$$
f\left(z_{1}, z_{2}\right)=8 z_{1}^{2}-7 z_{1} z_{2}-12 z_{1}-z_{2}+13
$$


First, the 1 -D condition $f\left(z_{1}, 0\right) \neq 0,\left|z_{1}\right| \leq 1$ is verified. Second, the condition $\mathrm{PE}_{f}(0)>\mathrm{PE}_{\bar{f}}(0)$ with $\left|z_{1}\right|=1$ or $\mathbf{a}_{(1)}^{t} \cdot \mathbf{T} \cdot \mathbf{x}=$ $119-518 x+416 x^{2}>0 \forall x$ with $-1 \leq x \leq 1$ is examined. The roots of the last polynomial are 0.3039 and 0.9413 , so for $x=0.5$ it is negative. Therefore, the corresponding 2-D system (1) is unstable. One can obtain that $f(0.4+0.8 i,-0.186026-0.904803 i)=0$, which assures the above result.

Example 3: Consider [15, Example 2].

$$
\begin{aligned}
f\left(z_{1}, z_{2}\right)= & {\left[\begin{array}{ccccc}
1 & z_{1} & z_{1}^{2} & z_{1}^{3}
\end{array}\right] } \\
& \cdot\left[\begin{array}{cccc}
1 & 0.1 & 0.25 & 0.1 \\
0.7 & 1.25 & 1.5 & 1.3 \\
-0.4 & -0.85 & -2 & 1.2 \\
-0.25 & 1.7 & -0.9 & 0.1
\end{array}\right]\left[\begin{array}{c}
1 \\
z_{2} \\
z_{2}^{2} \\
z_{2}^{3}
\end{array}\right] .
\end{aligned}
$$

The 1-D stability conditions are satisfied. By using $\mathrm{PE}_{f}(0)>\mathrm{PE}_{\bar{f}}(0)$ one finds $-1.4375-2.58 \cos \theta-1.65 \cos 2 \theta-0.52 \cos 3 \theta>0$ $\forall \theta, 0 \leq \theta \leq 2 \pi$, which directly fails for $\theta=0$. Thus, this 2-D system is unstable. The same result can be found by examining the equivalent inequality $\mathbf{a}_{(1)}^{t} \cdot \mathbf{T} \cdot \mathbf{x}=0.2125-1.02 x-3.3 x^{2}-2.08 x^{3}>0 \forall x$ with $-1 \leq x \leq 1$.

\section{CONCLUSION}

The minimal delay property gives useful necessary conditions for stability of a 1-D polynomial. This property can also supply us with useful necessary conditions for the stability of 2-D polynomials. A comparison with other necessary stability conditions is given via some illustrative examples.

\section{REFERENCES}

[1] E. I. Jury, "Stability of multidimensional systems and related problems," in Multidimensional Systems-Techniques and Applications, S. G. Tzafestas, Ed. New York: Dekker, 1985.

[2] S. J. Orfanidis, Optimum Signal Processing. New York: McGraw-Hill, 1990.

[3] N. J. Theodorou and S. G. Tzafestas, "Factorizability conditions for multidimensional polynomials," IEEE Trans. Automat. Contr., vol. AC-30, pp. 697-700, July 1985 .

[4] N. E. Mastorakis, N. J. Theodorou, and S. G. Tzafestas, "Factorization of m-D polynomials in linear m-D factors," Int. J. Syst. Sci., vol. 23, no. 11, pp. 1805-1824, 1992.

[5] N. E. Mastorakis, N. J. Theodorou, and S. G. Tzafestas, "A general factorization method for multivariable polynomials," Multidimensional Syst. Signal Processing, vol. 5, pp. 151-178, Apr. 1994.

[6] J. L. Shanks, S. Treital, and J. H. Justice, "Stability and synthesis of two dimensional recursive filters," IEEE Trans. Audio Electroacoust., vol. 20, pp. 115-208, June 1972.

[7] T. S. Huang, "Stability of two-dimensional recursive filters," IEEE Trans. Audio Electroacoust., vol. AU-20, pp. 158-163, June 1972.

[8] E. I. Jury, "Theory and applications of the inners," Proc. IEEE, vol. 63, pp. 1044-1069, July 1975.

[9] B. D. O. Anderson and E. I. Jury, "Stability test for two-dimensional recursive filters," IEEE Trans. Audio Electroacoust., vol. AU-21, pp. 366-372, Aug. 1972.

[10] D. D. Siljak, "Stability criteria for two-variable polynomials," IEEE Trans. Circuits Syst., vol. CAS-22, pp. 183-189, Mar. 1975.

[11] G. A. Maria and M. M. Fahmy, "On the stability of two-dimensional digital filters," IEEE Trans. Audio Electroacoust., vol. A\$-21, pp. 470-472, Oct. 1973.

[12] N. K. Bose and L. S. Kamat, "Algorithm for stability test of multidimensional filters," IEEE Trans. Acoust. Speech Signal Processing, vol. 22, pp. 307-314, Oct. 1974.

[13] N. K. Bose, "Implementation of a new stability test for two-dimensiona filters," IEEE Trans. Acoust. Speech Signal Processing, vol. 24, pp. 117-120, Apr. 1977.
[14] E. I. Jury and M. Mansour, "Positivity and nonnegativity conditions of a quartic equation and related problems," IEEE Trans. Automat. Contr., vol. AC-26, pp. 444-450, Apr. 1971.

[15] P. Agathoklis and M. Mansour, "Sufficient conditions for instability of two and higher dimensional discrete systems," IEEE Trans. Circuits Syst., vol. CAS-29, pp. 486-488, July 1982.

[16] D. Raghuramireddy and R. Unbehaven, "Stability of a class of 2-D recursive filters without distortion of the amplitude spectrum," IEEE Trans. Circuits Syst., vol. CAS-32, pp. 1296-1298, Dec. 1985.

[17] M. N. S. Swamy, L. M. Roytman, and N. Marinovic, "A necessary condition for the BIBO stability of 2-D filters," IEEE Trans. Circuits Syst. II, vol. 39, pp. 475-476, July 1992.

[18] N. E. Mastorakis, "Comments concerning multidimensional polynomials properties," IEEE Trans. Automat. Contr., vol. 41, p. 276, Feb. 1996.

[19] — "New stability test for 2-D systems," Foundation Comput. Decision Sci., vol. 21, no. 2, pp. 115-122, 1996.

[20] _ , "Recursive algorithms for two-dimensional filters' spectral transformations," IEEE Trans. Signal Processing, vol. 44, pp. 2647-2651, Oct. 1996.

[21] _ " "A method for computing the 2-D stability margin," IEEE Trans. Circuits Syst. II, vol. 45, pp. 376-379, Mar. 1998.

[22] — "The optimum radius of the robust stability of Schur polynomials," J. Optimization Theory Appl., vol. 96, no. 1, Jan. 2000

\section{Basic Synchronous Phenomena by Intermittently Coupled Capacitors}

Takanori Matsushita, Toshimichi Saito, and Hiroyuki Torikai

Abstract-We propose a simple coupling method using intermittently coupled capacitors (ICC's). We construct a coupling system by applying the ICC to two piecewise linear nonautonomous chaotic circuits. Then the ICC changes the two chaotic attractors into a coexisting state of chaos synchronization and periodic synchronization. The system exhibits one of them depending on the initial states. The system dynamics are reduced into a 3-D return map with one real and two binary variables and the occurence of the coexisting phenomenon is guaranteed theoretically. Also, typical phenomena are confirmed in the laboratory.

Index Terms-Chaos, mapping procedure, oscillator, synchronization, switched capacitor.

\section{INTRODUCTION}

The study of chaos synchronization is important not only as a basic nonlinear problem but also for new engineering applications, including artificial neural networks [1]-[3] and chaos-based communications [4], [5]. In the studies, there are two basic problems. First, the nonlinear system exhibits various kinds of synchronous phenomena, including phase synchronization [6], [7] and they should be classified

Manuscript received September 3, 1998; revised November 1, 1999. This paper was recommended by Associate Editor C. W. Wu.

T. Matsushita is with Oki Electric Industry Company Ltd., Tokyo 184, Japan (e-mail: matsushita@ coss.telcom.oki.co.jp).

T. Saito and H. Torikai are with the Department of Electronics and Electrical Engineering, Hosei University, Tokyo 184, Japan (e-mail: tsaito@k.hosei.ac.jp; torikai@k.hosei.ac.jp).

Publisher Item Identifier S 1057-7122(00)05502-1. 